\title{
Use of co-primary outcomes for trials of antimicrobial stewardship interventions
}

\section{Authors (no guidance on upper limit):}

David Gillespie ${ }^{1}$, Nick A Francis ${ }^{2}$, Enitan D Carrol ${ }^{3}$, Emma Thomas-Jones ${ }^{1}$, Christopher C Butler ${ }^{4}$, Kerenza Hood ${ }^{1}$

1 - Centre for Trials Research, College of Biomedical and Life Sciences, Cardiff University, Cardiff, U.K.

2 - Division of Population Medicine, School of Medicine, College of Biomedical and Life Sciences, Cardiff University, Cardiff, U.K.

3 - University of Liverpool Institute of Infection and Global Health, Liverpool, U.K.

4 - Nuffield Department of Primary Care Health Sciences, University of Oxford, Oxford, U.K.

Target journal: Lancet Infectious Diseases

Type of article: Comment (aim for up to 750 words and maximum ten references - no separate sections)

Manuscript text: Currently 743/750 words

Antimicrobial resistance (AMR) is a major public health threat that will cause an estimated 10 million deaths worldwide by 2050. [1] Because antimicrobial use drives selection and transmission of AMR, $[2,3]$ there is an urgent need to continue to develop, evaluate, and implement effective, evidencebased antimicrobial stewardship (AMS) interventions that safely reduce antimicrobial use in both primary and secondary healthcare. $[4,5]$

AMS interventions can benefit individual patients, but are often viewed as trading potential increased short-term individual risk for long-term societal gain, [6] and so some consider them 'bedside rationing'. [7] The need to consider the ethical implications of AMS programmes, and acceptable trade-offs, will increase as antimicrobial prescribing is reduced and programmes are investigated among patients with an increased chance of benefit from antimicrobials.

While there is a clear need to ensure that reductions in antimicrobial use are not at the expense of patient outcomes, most current research considers these outcomes separately. AMS studies are therefore generally powered on only one of these aspects (typically antimicrobial use), and as a result, are frequently underpowered to detect clinically meaningful differences in important clinical secondary outcomes, and do not pre-specify whether between-group differences in clinical outcomes will be investigated under superiority, non-inferiority, or equivalence hypotheses.

This article describes the use of co-primary outcomes as a solution to this problem, presents two examples of co-primary outcomes in AMS intervention trials, and argues for their routine use in AMS intervention evaluations.

Co-primary outcomes involve the use of two or more primary outcomes, and rejecting both null hypotheses is necessary for the intervention to be declared successful. This contrasts with studies that have multiple primary outcomes where rejecting the null hypothesis for at least one (i.e. alternative primary outcomes) determines success. The use of co-primary outcomes in AMS intervention research encourages researchers to pay close attention to both antimicrobial use and patient-relevant measures (e.g. recovery from illness, safety outcomes), ensuring the study is adequately designed to simultaneously answer both questions. Thus, any reduction in antibiotic use must be judged in conjunction with any negative impact on patient recovery. Additional sample size considerations for 
trials with co-primary outcomes include the assumed correlation between outcomes and the overall power of the study. When outcomes are completely independent, the overall power (to detect similar effect sizes in both outcomes) is the product of the power for each individual outcome, and only when they are perfectly correlated is the overall power unaffected. [8]

The PACE determined the effectiveness of C-reactive Protein (CRP) point-of-care testing on safely reducing antibiotic use in patients presenting in primary care with an acute exacerbation of chronic obstructive pulmonary disease (AECOPD). [9] The study used two co-primary outcomes: i.) Antibiotic use for AECOPD within the first four-weeks post-randomisation; ii.) COPD health status, as measured by the COPD Clinical Questionnaire at two-weeks post-randomisation. These co-primary outcomes were investigated for superiority and non-inferiority respectively. The study aimed to recruit 650 participants in order to achieve between $81 \%$ and $90 \%$ power to detect a $20 \%$ absolute difference in antibiotic use, and a COPD health status that is no worse (with a non-inferiority margin of 0.3 ).

The BATCH study is investigating the effect of procalcitonin-guided management on antibiotic use in children with severe bacterial infection. [10] The co-primary outcomes for the study are i.) Number of days of intravenous (IV) antibiotic therapy, and; ii.) Safety (comprising unscheduled admissions/readmissions; re-treatment for same condition within 7 days of stopping IV antibiotics; mortality). The study aims to recruit 1942 participants to achieve between $99 \%$ power to detect a decrease in antibiotic duration and $90 \%$ power to test non-inferiority in safety. Assuming that the antibiotic use and safety primary outcomes are independent, this will give at least $89 \%$ power for the combined analysis.

Reducing antimicrobial use is essential to preserving antimicrobial effectiveness, but should not harm patients in the process. AMS intervention evaluations must consider the clinical implications of changed antimicrobial use, and the use of co-primary outcomes ensures that both use and safety outcomes are considered jointly. Researchers in this field may therefore wish to consider the use of co-primary outcomes, and research funders may consider mandating this dual focus when commissioning AMS intervention studies.

\section{Acknowledgements}

The authors declare no competing interests. The PACE and BATCH studies were funded by the National Institute of Health Research Health Technology Assessment (PACE: HTA project reference 12/33/12 ISRCTN 24346473; BATCH: HTA project reference 15/188/42 ISRCTN 11369832). The work was undertaken with the support of the UKCRC registered Centre for Trials Research. Infrastructure funding from Health and Care Research Wales and Cancer Research UK is gratefully acknowledged. CCB is an NIHR Senior Investigator and Director of the Primary Care and Vaccines Clinical Trials Cooperative and the NIHR Community Medical Technology and Invitro-diagnostics Cooperative, University of Oxford.

\section{References}

1. World Health Organization, 2014. Antimicrobial resistance: global report on surveillance. World Health Organization.

2. Goossens, H., Ferech, M., Vander Stichele, R., et al. Outpatient antibiotic use in Europe and association with resistance: a cross-national database study. The Lancet, 365(9459), pp.579-587. 
3. Costelloe, C., Metcalfe, C., Lovering, A., et al, 2010. Effect of antibiotic prescribing in primary care on antimicrobial resistance in individual patients: systematic review and meta-analysis. Bmj, 340, p.c2096.

4. Davey, P., Marwick, C.A., Scott, C.L., et al, 2017. Interventions to improve antibiotic prescribing practices for hospital inpatients. The Cochrane Library.

5. Aldeyab, M.A., Scott, M.G., Kearney, M.P., et al, 2014. Impact of an enhanced antibiotic stewardship on reducing methicillin-resistant Staphylococcus aureus in primary and secondary healthcare settings. Epidemiology \& Infection, 142(3), pp.494-500.

6. Cosby, J.L., Francis, N. and Butler, C.C., 2007. The role of evidence in the decline of antibiotic use for common respiratory infections in primary care. The Lancet infectious diseases, 7(11), pp.749-756.

7. Oczkowski, S., 2017. Antimicrobial stewardship programmes: bedside rationing by another name?. Journal of Medical Ethics, pp.medethics-2015.

8. Offen, W., Chuang-Stein, C., Dmitrienko, A., et al, 2007. Multiple co-primary endpoints: medical and statistical solutions: a report from the multiple endpoints expert team of the Pharmaceutical Research and Manufacturers of America. Drug Information Journal, 41(1), pp.31-46.

9. Bates, J., Francis, N.A., White, P., et al, 2017. General practitioner use of a C-reactive protein pointof-care test to help target antibiotic prescribing in patients with acute exacerbations of chronic obstructive pulmonary disease (the PACE study): study protocol for a randomised controlled trial. Trials, 18.

10. NIHR HTA. 2017. Biomarker-guided duration of Antibiotic Treatment in Children Hospitalised with confirmed or suspected bacterial infection (The BATCH Trial). [ONLINE] Available at: https://www.journalslibrary.nihr.ac.uk/programmes/hta/1518842/\#/. [Accessed 24 January 2018] 